\title{
LES « BRÛLEURS » DE FRONTIÈRES DANS LA MUSIQUE POPULAIRE TUNISIENNE
}

La migration non documentée au prisme de chansons de rap et de mezoued Monika Salzbrunn, Farida Souiah, Simon Mastrangelo

De Boeck Supérieur | « Afrique contemporaine »

2015/2 n $254 \mid$ pages 37 à 56

ISSN 0002-0478

ISBN 9782807300743

Article disponible en ligne à l'adresse :

http://www.cairn.info/revue-afrique-contemporaine-2015-2-page-37.htm

\section{!Pour citer cet article :}

Monika Salzbrunn et al., «Les «brûleurs » de frontières dans la musique populaire tunisienne. La migration non documentée au prisme de chansons de rap et de mezoued », Afrique contemporaine 2015/2 ( $\left.{ }^{\circ} 254\right)$, p. 37-56.

Distribution électronique Cairn.info pour De Boeck Supérieur.

(C) De Boeck Supérieur. Tous droits réservés pour tous pays.

La reproduction ou représentation de cet article, notamment par photocopie, n'est autorisée que dans les limites des conditions générales d'utilisation du site ou, le cas échéant, des conditions générales de la licence souscrite par votre établissement. Toute autre reproduction ou représentation, en tout ou partie, sous quelque forme et de quelque manière que ce soit, est interdite sauf accord préalable et écrit de l'éditeur, en dehors des cas prévus par la législation en vigueur en France. Il est précisé que son stockage dans une base de données est également interdit. 


\title{
Les « brûleurs " de frontières dans la musique populaire tunisienne
} La migration non documentée au prisme de chansons de rap et de mezoued

\author{
Monika Salzbrunn \\ Farida Souiah \\ Simon Mastrangelo
}

\begin{abstract}
Cet article propose une analyse des représentations des « brûleurs " de frontières, les migrants d'Afrique du Nord, dans la musique populaire tunisienne à partir d'un corpus constitué de cinquante-quatre chansons. II mobilise la musique populaire tel un révélateur de l'imaginaire migratoire et de dynamiques sociales profondes. Après avoir exploré le contexte d'énonciation des chansons, l'article se structure autour des quatre thèmes prévalents: désirs migratoires, dangers de la traversée, souffrance de l'exilé et des siens, acceptation de la volonté divine. Cette analyse permet de conclure que, sans pouvoir être classées dans la musique révolutionnaire au sens classique du terme, les paroles des chansons sur la harga contiennent un potentiel de subversion important: voter avec ses pieds.
\end{abstract}

Mots clés : Migration non documentée - Harraga - Harga - Musique - Tunisie - Rap - Europe - Chansons Frontières - Imaginaires - Représentation

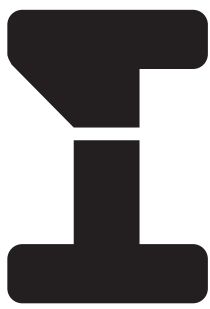

« Ils sont partis » : c'est par ce constat que se clôt l'une des plus célèbres chansons tunisiennes sur la harga. Dans ces quelques vers, Balti et Samir Loussif évoquent les risques de la traversée par la mer, ainsi que la douleur des personnes que les migrants laissent derrière eux. Au Maghreb, le mot harga, littéralement l'acte de «brûler », désigne un phénomène migratoire. Les harraga, les «brûleurs », sont ceux qui tentent de quitter
Monika Salzbrunn est professeure en «Religions, Migration, Diasporas» à l'université de Lausanne, directrice de l'Institut des sciences sociales des religions contemporaines (ISSRC) et de l'Observatoire des religions en Suisse (ORS).

Farida Souiah et Simon

Mastrangelo sont respectivement post-doctorante et doctorant dans le cadre du projet « Undocumented Mobility (Tunisia-Switzerland) and
Digital-Cultural Resources after the "Arab Spring" ", dirigé par Monika Salzbrunn et financé par le Fonds national suisse de la recherche scientifique. 
leur pays sans passeport ni visa, au péril de leur vie. On les nomme ainsi, car ils «brûlent » les frontières, ainsi que les étapes nécessaires à un départ qui respecterait les contraintes imposées par les États. En outre, s’ils arrivent en Europe, ils détruisent, «brûlent » littéralement leurs papiers d'identité, pour échapper à l'expulsion.

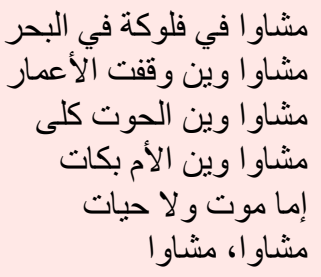

Ils sont partis en barque sur la mer

Ils sont partis là où les vies s'arrêtent

Ils sont partis là où le poisson mange [les hommes]

Ils sont partis là où la mère pleure

Sont-ils vivants ou morts?

Ils sont partis, partis ${ }^{1}$.

Le phénomène de la harga, qui s'est développé à partir des années 1980, est lié à la mise en place de politiques migratoires restrictives en Méditerranée (Düvell, 2008 ; MPC Team, 2013 ; Souiah, 2013) et à la diminution des possibilités de circuler entre les deux continents européens et africains. Les contraintes à la mobilité touchent de façon asymétrique les populations. Alors que certains ont le droit à la mobilité du fait de leur revenu, de leur niveau de formation ou, encore, un passeport européen, d'autres sont privés de ce droit, car ils sont perçus comme de potentiels migrants « indésirables » (Agier, 2008). Les harraga sont, pour la plupart, des jeunes hommes célibataires sans emploi suffisamment stable ou rémunéré (Boubakri, 2013 ; Labdelaoui, 2009 ; Mabrouk, 2010 ; Rouis, 2008) pour leur permettre de prétendre à un visa pour l'espace Schengen ${ }^{2}$. Ils sont de facto exclus du système de mobilité légale, c'est pourquoi ils tentent de «brûler» les frontières. Les politiques migratoires

1. Balti et Samir Loussif, «Mchaou » (« lls sont partis »), 2011.

2. II y a plusieurs voies de migration, au sens propre comme au sens figuré (notamment selon les différents types de visa qui permettent de se déplacer dans le cadre d'un système juridique établi). Le phénomène de la harga s'est amplifié au fur et à mesure que les voies légales de la migration ont été restreintes (en diminuant le nombre de visa et en rendant les conditions d'obtention de plus en plus difficiles) (Wihtol de Wenden, 2013).

3. Démarche notamment adoptée par Farida Souiah dans ses travaux antérieurs sur la harga dans la musique algérienne (Souiah, 2011, 2014). 
restrictives ne font pas naître le désir d'émigrer, mais elles peuvent le nourrir et elles influencent grandement les modalités de départ (Souiah, 2014). Or, les États de la rive nord de la Méditerranée choisissent de combattre les flux migratoires «irréguliers » en mettant en œuvre des politiques toujours plus restrictives et sécuritaires (Wihtol de Wenden, 2010 et 2013 ; Migreurop, 2012). De façon croissante, les États de la rive sud prennent part à cette lutte (Bensaâd, 2009 ; El Qadim, 2013 ; Natter, 2014). La signature de différents accords de collaboration entre les États maghrébins, l'Union européenne ou les États qui la composent, n'a pas mené à une diminution des flux migratoires non documentés (Wihtol de Wenden, 2013). Ceci révèle le «paradoxe libéral » (Hollifield, 1992) qui permet aux marchandises et aux finances de circuler de plus en plus librement, tandis que les migrants potentiels, privés de ces droits de circuler à cause du renforcement des frontières européennes, sont amenés à trouver d'autres solutions. Ainsi, des stratégies de dépassement des frontières de manière concrète et de manière symbolique émergent. La musique fait partie de cette inversion symbolique du pouvoir.

« Avant d'être sujet quasi quotidien de reportage dans la presse et les médias, avant d'être sujet d'inquiétude et de focalisation institutionnelle, la harga est d'abord un sujet d'inspiration pour des artistes [...] » (Peraldi et al., 2014, p. 143). Les récits et les mots des productions culturelles se distinguent des discours politiques et médiatiques sur la migration puisqu'on y voit des migrant(e-s) comme acteur(e-s) et narrateur(e-s) de leur vie (Canut, Sow, 2014 ; Salzbrunn 2008). Denis-Constant Martin affirme que les «pratiques culturelles observables » et les « œuvres issues de certaines d'entre elles » (2003, p. 21) permettent d'approcher la culture, l'imaginaire et le symbolique. Elles sont une porte d'entrée sur le «politique au style indirect » et exposent le pouvoir dans son ambiguïté et son ambivalence. D’après ce chercheur, la musique est un moyen privilégié d'appréhender le politique : c'est «par les astuces du double langage, de l'évocation codée, dans les malices de la métaphore et les ruses du symbolique que, le plus souvent, se sont faites entendre les voix critiques » (ibid., p. 2). En outre, en raison de l'abondance de sa création, de son impact et de sa diffusion, « la chanson est, au Maghreb, le mode privilégié de l'expression culturelle»(Virolle, 1995, p. 6). Les paroles des chansons reflètent parfois une manière de tourner en dérision l'image stigmatisante apposée à la migration non documentée (Salzbrunn, 2012, p. 6-7). De son côté, Heidrun Friese (2015) évoque l’importance de l'imaginaire - qui s'exprime notamment par la musique - dans l'émergence du désir de migrer. Ainsi, la harga comme thème de chansons est l'expression d'un projet migratoire observé (par les auteur[e-s] des paroles), mais parfois aussi d'un désir propre à celles et ceux qui interprètent ces chansons. Puis, la circulation de ces chansons sur Internet nourrit l'imaginaire migratoire des harraga. Ainsi, l'analyse de la musique a ici vocation à éclairer les représentations de la harga et l'imaginaire migratoire des Tunisiens. Cette démarche permet de saisir les représentations « au ras du sol $»^{3}$. 
De quoi traitent ces chansons de rap et de mezoued tunisiennes sur la harga? Que nous apprennent-elles de l'imaginaire migratoire tunisien? Quelles représentations de la Tunisie et de l'Europe participent-elles à construire et à véhiculer (Salzbrunn, 2012 ; Friese, 2012) ? Quelles facettes de la vie en Tunisie ces chansons mettent-elles en avant pour expliquer le désir migratoire ? Quelles représentations de la «bonne vie » en Europe véhiculentelles et comment abordent-elles la traversée de la Méditerranée et le danger mortel qu'elle implique (Salzbrunn, 2012, p. 10-11) ? Quels rôles ces chansons attribuent-elles à la foi et à la religion dans le parcours des migrants, notamment dans la manière dont ils appréhendent la prise de risque ?

Les chansons sur la harga sont un objet d'étude d'autant plus prometteur qu'elles n'entrent pas facilement dans les catégories établies dans le courant des travaux portant sur musique et migration (Martiniello, Lafleur, 2008) ou musique et action communautaire : action politique confrontative, action politique délibérative et action politique pragmatique (Mattern, 1998). Il n'est pas non plus facile de cataloguer ces chansons selon les catégories d'analyse proposées par Ian Peddie (2012) ou par Ian Biddle (2012), éditeurs d'encyclopédies récentes sur musique et protestation et sur musique et politiques identitaires. La musique sur la harga devient-elle « révolutionnaire » par l'importance que lui donnent les harraga (Mattern, 1998) ? Selon Ian Peddie, la musique est toujours produite socialement, puis «music is made political or revolutionary » (2012, p. XIII). L'acte de l'écouter devient-il ainsi une échappatoire pour celles et ceux qui se sentent dépossédés de leur parole ${ }^{4}$ ? Les termes proposés récemment par Lionel Arnaud semblent plus appropriés pour le cas des harraga : l'expression des désirs et de la souffrance ou la projection dans une vie en dehors de la Tunisie ne reviendraient-elles pas à « maîtriser symboliquement le monde » (2014, p. 4)?

Malgré la diversité des perspectives - les interprètes incarnent parfois des harraga, d'autres fois des membres de la famille de harraga ou une personne tierce qui raconte l'histoire d'un « brûleur » de frontières -, certains thèmes et champs lexicaux ${ }^{5}$ prévalent dans les chansons sur la harga. Après avoir exploré

4. Biddle les appelle «the similarly dispossessed " (Biddle, 2012, p. XI-XXIV). Par ailleurs, les migrants au sens large et les harraga en particulier n'ont pas toujours été considérés comme des acteurs par une majeure partie des chercheurs en sciences sociales (Salzbrunn, 2008, p. 75-100). L'étude des créations artistiques dans un contexte migratoire est encore plus récente.

5. La marginalité socio-économique, l'oppression, la souffrance, la mort, l'exil, la réussite comptent parmi les thèmes et les champs lexicaux développés dans ces chansons.
6. Selon les données de l'agence Frontex, 20258 Tunisiens seraient arrivés sur l'île de Lampedusa, entre janvier et mars 2011 (Boubakri, 2013).

7. Au total, 289 vidéos ont été postées sur les vingt pages Facebook étudiées. Parmi elles, 190 ont été publiées entre 2011 et 2012 (soit plus de $65 \%$ du total des vidéos de notre corpus).

8. Parmi elles, quarante-six traitent spécifiquement du thème de la harga, cinq traitent de la migration ou de l'exil de façon plus générale et trois traitent de sous-thèmes voisins, tels que celui de la dureté de la vie en
Tunisie ou du deuil. Le corpus a été constitué par l'équipe et les chansons ont été transcrites et traduites par Farida Souiah.

9. Cet instrument, le mezoued, qui donne son nom au style musical, lui confère une sonorité caractéristique et facilement reconnaissable.

10. Il est difficile de déterminer avec exactitude les origines du mezoued. Ce genre est né avec l'arrivée de migrants issus de zones rurales dans les milieux urbains, en particulier à Tunis, au milieu du $x x^{e}$ siècle (Stapley, 2006). 
le contexte d'énonciation, notre article se structurera autour des quatre thèmes principaux des chansons : les désirs migratoires, les dangers de la traversée, la souffrance de l'exilé et des siens, ainsi que l'acceptation de la volonté divine.

\section{Migration et harga : des sujets incontournables de la musique populaire tunisienne}

Débuté en 2010, un mouvement de contestation a provoqué la chute du régime de Ben Ali, le 14 janvier 2011 (Hibou, 2011). À la suite de ces événements, le pays a vécu une période durant laquelle la police a quitté les espaces publics, les commissariats se sont vidés et la surveillance des côtes, menée en collaboration avec les autorités européennes, n'a plus été effectuée (Boubakri, 2013). Ce retrait a rendu possible le départ, très médiatisé tant au niveau national qu'international, d'un nombre important de harraga, en particulier entre janvier et mars $2011^{6}$. Cet engouement pour la harga s'est notamment traduit par la création de nombreuses pages Facebook sur cette thématique en 2011 et $2012^{7}$. Dès le mois d'avril 2011, le nombre de départs par harga a commencé à diminuer, notamment à cause de la reprise en main de la surveillance des côtes tunisiennes (Boubakri, 2013). Par la suite, le thème de la harga a été de façon progressive de moins en moins médiatisé et le nombre de vidéos a diminué.

Afin de constituer leur corpus, les auteurs se sont fondés sur les contenus musicaux partagés sur vingt pages Facebook tunisiennes dédiées à la harga dont quatorze ont été créées entre 2011 et 2012 (sept en 2011 et sept en 2012). Sur ces pages, des Tunisiens publient des clips musicaux et des diaporamas qu'ils créent grâce au montage d'images qu'ils collectent sur Internet et qu'ils associent à des chansons. Le choix de constituer un corpus en partant des réseaux sociaux permet d'inclure des chanteurs dont le degré de professionnalisation est varié. En outre, cela montre que les Tunisiens qui désirent migrer s'approprient ces chansons dont les paroles résonnent avec leur imaginaire ou leur expérience migratoire. Les pages Facebook sur la harga nous ont permis de constituer un corpus de cinquante-quatre chansons ${ }^{8}$ tunisiennes dont trentesix ont été mises en ligne entre 2011 et 2012.

En Tunisie, la figure du «brûleur » de frontières ne suscite pas un intérêt égal au sein des différents genres musicaux. Elle apparaît essentiellement dans le rap et le mezoued. Ce dernier genre musical, dont l'instrument principal est similaire à une cornemuse ${ }^{9}$, généralement accompagné par du chant et des percussions, n'a été que peu diffusé par les canaux médiatiques officiels ${ }^{10}$ jusqu'aux années 1970. Le mezoued était « associé à des groupes marginalisés dans la société tunisienne » (Stapley, 2006, p. 245) et avait mauvaise réputation. C'est il y a une quarantaine d'années (avec les premiers enregistrements), puis surtout à partir des années 1990 et une progressive médiatisation, que le genre s'est popularisé et que son image a évolué (Stapley, 2006). Les chansons de mezoued évoquent notamment les difficultés de vie rencontrées dans les quartiers populaires. Parmi les thèmes principaux abordés, il y a à la fois la 
migration depuis la campagne vers les zones urbaines, la migration internationale, ainsi que les difficultés matérielles et émotionnelles rencontrées par les migrants (Stapley, 2006).

Le genre musical dominant au sein de ce corpus n'est cependant pas le mezoued, mais le rap. En Tunisie, bien que peu médiatisée et marginalisée, la culture hip-hop a commencé à se développer à partir de la fin des années 1980 avec quelques précurseurs dans des milieux très restreints. Internet a contribué à populariser ce genre musical (Frikha, 2009). Sous le régime de Ben Ali, la liberté d'expression ayant été considérablement limitée (Hibou, 2005), certains rappeurs s'autocensuraient (Shannahan et Hussain, 2010, p. 44). Cette situation a commencé à changer autour de 2010 avec des chansons critiques envers le régime par des rappeurs politisés comme Psyco-M et El Général. Une chanson de ce dernier, intitulée « Rais lebled» («Le chef du pays »), a eu un impact particulièrement important, surtout au niveau symbolique. Dans cette chanson, qui a réussi à se propager, malgré la censure, via les réseaux sociaux, El Général critique ouvertement le régime de Ben Ali. S’adressant directement à l'ex-président, il dénonce la corruption et l'intimidation dont est victime le peuple tunisien (Gana, 2012). À la suite d'El Général, d'autres rappeurs tunisiens ont décidé de s'engager plus ouvertement.

\section{La dureté de la vie en Tunisie}

Les chansons du corpus, dont la majorité est écrite dans la perspective d'une personne qui désire «brûler» les frontières, abordent les raisons pour lesquelles les jeunes veulent «fuir » le pays. Ces vers et ces couplets, consacrés aux causes du départ, révèlent que l'envie de «brûler » les frontières est fortement liée au quotidien des jeunes des classes populaires en Tunisie. Alors qu'elles sont le fruit de l'écriture de nombreux artistes, elles relèvent d'un imaginaire relativement homogène, utilisant des figures, des leitmotivs et des champs lexicaux similaires. Il existe un grand nombre de points communs dans les portraits que ces chansons dressent des harraga. Quel que soit leur niveau d'éducation, ils sont présentés comme des chômeurs ${ }^{11}$. À ce titre, le champ lexical du chômage est très présent, de même que celui des difficultés économiques et de la

11. Selon l'Institut national des statistiques (INS) tunisien, le taux de chômage en 2014 est de $15,3 \%$ (www.ins.nat.tn/). Ce chiffre doit être pris avec précaution, car seules les personnes qui ne travaillent pas, mais effectuent des démarches afin de trouver un emploi formel, sont considérées comme chômeuses. La plupart des harraga rencontrés sur le terrain n'entreraient pas dans cette catégorie, car les statistiques officielles les considèrent comme des personnes inactives.

12. Ali Dji et Amine, «Machi », 2015.

13. El Mister, "Vue noire », 2012.

14. Afin de transcrire les titres des chansons en dialecte tunisien, nous avons mobilisé les équivalences numériques qui sont utilisées au Maghreb et au Moyen-Orient pour écrire les dialectes en alphabet latin sur Internet et dans les messages téléphoniques. Les chiffres

permettent de retranscrire des lettres arabes qui n'ont pas d'« équivalent graphématique dans l'écriture latine » (Saadane et al., 2013, p. 6). Ce choix a été fait afin de faciliter la recherche des chansons sur des plateformes telles que YouTube pour les lecteurs qui souhaiteraient les écouter.

15. DJ Costa fait une référence à un proverbe maghrébin sur la harga sur lequel les auteurs reviendront ultérieurement.

16. AKM, «9assed rabi » ("Je m'en remets à Dieu »), 2012. 
pauvreté ( فر ). Les harraga se sentent prisonniers de la situation socio-économique dans laquelle ils se trouvent et décrivent un univers où les emplois sont réservés à ceux qui détiennent du capital social.

Ce sentiment d'être contraint se matérialise notamment à travers l'évocation fréquente des murs (حيوط) dans les paroles. Ce sont les murs au sens littéral, ceux de leurs quartiers auxquels ils vivent adossés, car ils n'ont pas de travail. Ce sont également les murs au sens figuré, soit les obstacles infranchissables et les refus auxquels ils sont confrontés, notamment ceux des employeurs potentiels. Parfois, les critiques de la situation socio-économique en Tunisie se font plus explicitement politiques. C'est le cas dans certaines des chansons plus récentes qui présentent la révolution tel un échec. Ali Dji et Amine déclarent que la révolution n'a été qu'une plaisanterie et n'a fait qu'empirer la situation : davantage de mensonges et de vol au vu et au su de tout le monde ${ }^{12}$.

Les harraga sont présentés telles des victimes. Le harrag-type qui est décrit dans ces chansons a beaucoup de problèmes (مهوم) et il est victime de l'injustice et de l'oppression (مظلوم). La conviction d'être une victime du système socio-économique et politique est à l'origine d'un véritable mal-être qui est exprimé à travers la prévalence des champs lexicaux de la douleur, de la souffrance et des pleurs. Dans «Vue noire », El Mister rappe dans la perspective d'un «brûleur » de frontières. Dans le refrain qui ressemble à un cri, il dit qu'il a l'impression d'étouffer (مخنوق) en Tunisie et que la vie y est amère (مرّة) et courte (قصيرة). Après avoir été arrêté sur une embarcation, il est interrogé par un juge qui l'humilie et lui demande pourquoi il a tenté de «brûler ». Il parle alors de son quotidien en Tunisie. À 26 ans, il est convaincu que sa vie n'a été qu'une pure perte : son diplôme n'a aucune valeur, ses poches sont toujours vides et il doit demander à son père la somme nécessaire pour s'acheter des cigarettes. Il vit adossé à un mur avec la sensation d'être déjà mort ${ }^{13}$ qui est évoquée dans de nombreuses chansons. Ainsi, dans « 7oudoud $»^{14}$ (« Frontières »), DJ Costa compare le pays à une morgue où les jeunes se font manger par les vers $^{15}$. Cette impression de ne pas avoir d'avenir et de perspectives dans le pays fait d'eux des personnes excédées qui se sentent incapables de supporter la vie en Tunisie. Ainsi, des expressions comme «ça suffit » ou «j'en ai assez » sont très fréquemment mobilisées. Les « jeunes » protagonistes des chansons sur la harga ne veulent plus endurer cette vie et envisagent la harga comme l'unique solution qui s'offre à eux.

\section{L'Eldorado européen}

Bien que le quotidien difficile des jeunes de quartiers populaires soit traité de façon plus extensive que leurs attentes vis-à-vis de l'Europe et des pays européens, il est possible de voir s'esquisser les traits d'un Eldorado européen à traits plus ou moins épais dans ce corpus. Partir, c'est avant tout tenter sa chance et être mû par ses rêves, selon les paroles de chansons sur la harga. Dans de nombreux cas, les harraga aspirent à une «vie paisible ») 1(العيشة الهانية) 
ou veulent renouer avec le sentiment de joie qu'ils semblent incapables de ressentir en Tunisie ${ }^{17}$. Dans certaines chansons, les vers dédiés au pays ou à la ville de destination ${ }^{18}$ prennent la forme d'une déclaration d'amour recourant à la personnification. C'est le cas de la chanson « Ya Roma » (« Ô Rome ») dont sont tirés les quelques vers ci-dessous.

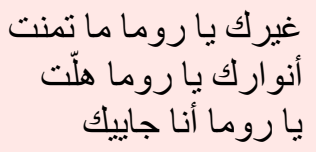

Je ne veux que toi, ô Rome

Ô Rome, tes lumières apparaissent

Ô Rome, nous arrivons ${ }^{19}$.

Les évocations des attentes des harraga vis-à-vis de l'Europe ont, cependant, rarement cette tonalité romantique, car elles se focalisent, entre autres, sur des ambitions matérialistes. À ce titre, le champ lexical de l'argent ${ }^{20}$ est particulièrement présent. Les gains ne sont pas uniquement destinés au harrag et à sa vie en Europe. De retour au pays, il s'agit de pouvoir faire acte de générosité, afin de projeter une image du succès, mais également d'améliorer le quotidien des siens. Outre l'argent, c'est la voiture qui concentre les ambitions des harraga. Revenir au pays avec un véhicule immatriculé en Europe est le symbole par excellence de la réussite. D’ailleurs, le mot «matricule », en français, peut être utilisé en dialecte pour parler d'une voiture apportée de l'étranger. Les ambitions des harraga sont parfois liées au désir de suivre l'exemple des migrants ${ }^{21}$ qui les ont précédés, comme l'illustre la chanson «Mchaou » (« Ils sont partis ») de Balti et Samir Loussif ci-dessous.

17. DJ Costa, « Hani machi »

(«Je pars»), 2011.

18. Alors que certaines chansons ne spécifient pas la destination des harraga, d'autres mettent en avant des espaces géographiques plus ou moins précis tels que "Bled el-ghir» ("Le pays des autres »), "Bled el-gaouri » ou «Bled el-roum» ("Le pays occidental»). La France et l'Italie qui sont évoquées lorsque des destinations précises sont mentionnées. L'importance donnée à ces destinations est liée à l'histoire migratoire de la Tunisie. Ce sont les deux principales destinations de la migration tunisienne (Di Bartolomeo et al., 2010). En outre, I'Italie est le lieu de transit le plus accessible des migrations par la mer au départ de la Tunisie en raison de sa proximité géographique.

19. Cheb Rached, "Ya Roma »

("Ô Rome »), 2011.

20. Ils mobilisent le mot « argent » en arabe (فلوس), en anglais dans le texte (money), des monnaies spécifiques (euros et dollars) et des expressions familières qui désignent le gain pécunaire telles que «se remplir les poches".
21. Selon l'Observatoire des Tunisiens à l'étranger (OTE), 1223213 Tunisiens résident à l'étranger en 2012, dont 84,4\% en Europe. Cela représente environ $10 \%$ de la population tunisienne. Ces données doivent être prises avec précaution, car elles n'incluent pas les migrations non documentées. 22. Le verbe جرب peut également se traduire par "faire l'expérience » ou " essayer».

23. Balti et Samir Loussif, "Mchaou » ("Ils sont partis »), 2011.

24. Baster et Malek, «Wa3di »

("Ma promesse »), 2012. 


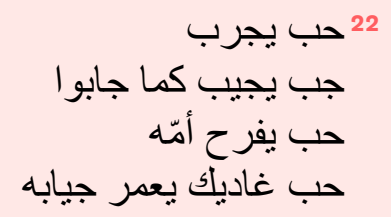

Il voulait tenter sa chance

Il voulait amener [de l'argent] comme les autres l'ont fait

Il voulait rendre heureuse sa mère

Il voulait remplir ses poches là-bas ${ }^{23}$.

\section{Les dangers de la traversée et l'issue funeste de l'aventure migratoire}

Hormis les causes du départ, les chansons sur la harga traitent des dangers de la traversée et de son éventuelle issue funeste. Parfois, les risques sont évoqués afin d'exprimer l'acceptation de ceux-ci. Dans la chanson «Wa3di » (« Ma promesse »), le protagoniste affirme qu'il est prêt à se confronter aux vagues et que le risque de mourir ne le dissuade pas : « Soit nous arriverons sains et saufs, soit nous serons mangés par les poissons ${ }^{24}$. » Cette référence aux corps des migrants qui sont abandonnés à la mer et nourrissent les poissons est courante, car elle reprend une expression popularisée au Maghreb depuis que le phénomène migratoire des harraga a pris de l'ampleur : «Que des poissons me mangent plutôt que des vers» (يكولني الحوت وما ياكونيش الدود). Cette expression est une mise en avant de l'acceptation des risques et plus spécifiquement de l'issue

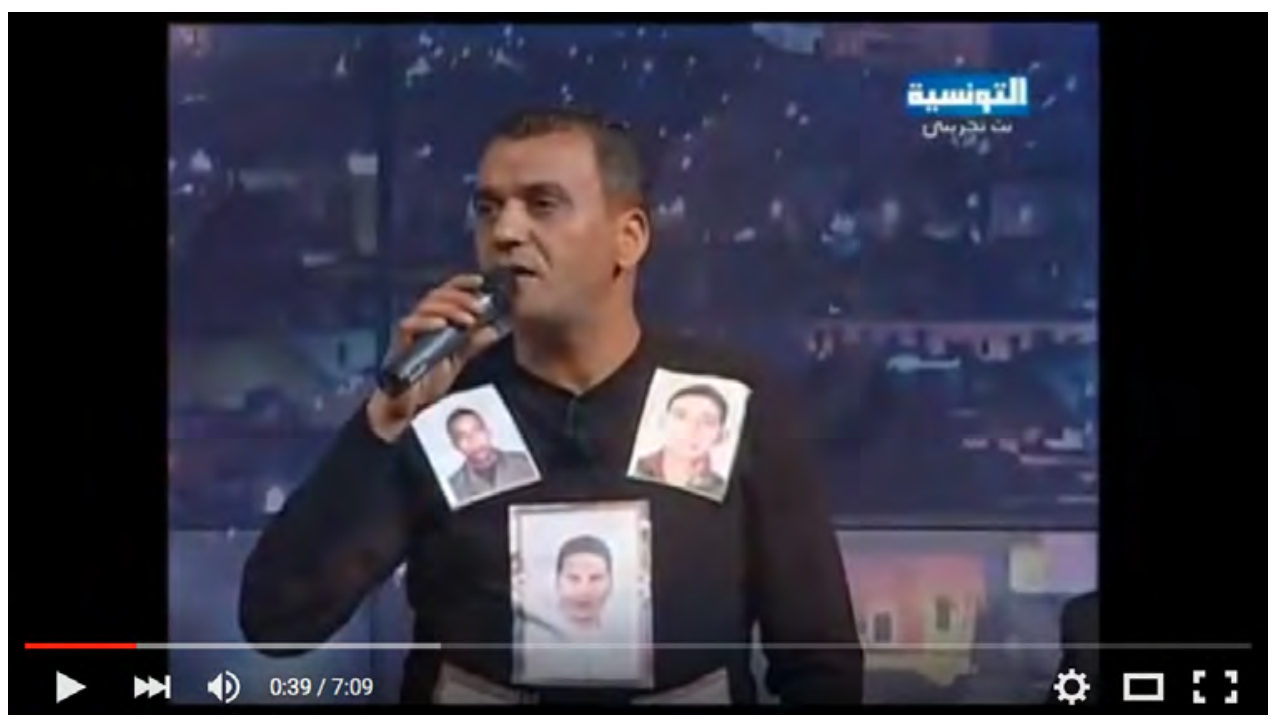

"Ya Roma ». Le chanteur de mezoued Nabil Louhichi interprète la chanson "Ya Roma » (2012) sur un plateau de télévision tunisien. Geste symboliquement fort, il a accroché des photos de harraga disparus en mer sur le haut de ses vêtements. Photo prise en capture d'écran sur YouTube : www.youtube.com/watch?v=eWZvC1IAnK4, 2016. 
funeste de l'aventure migratoire qui peut priver le migrant et sa famille d'un véritable rite funéraire. Comme cela fut évoqué dans la partie consacrée à la dureté de la vie en Tunisie, certains harraga ont la sensation d'être déjà morts.

Dans d'autres chansons, la prise de risque n'est pas seulement acceptée, mais valorisée, car elle permet aux harraga de manifester leur courage. En outre, elle symbolise une reprise de contrôle. Ils refusent de rester dans un pays où ils se sentent marginalisés et transgressent les lois qui régulent la circulation en Méditerranée et qui les excluent du système de mobilité légale. Le harrag qu'incarne Cheb Rached dans «Ya Roma » affirme que les dangers de la traversée ne sont pas dissuasifs.

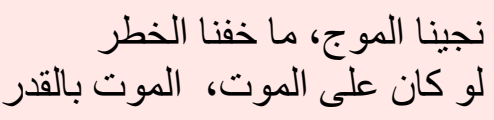

Nous nous sommes livrés aux vagues, nous n'avons pas eu peur du danger Si l'on doit mourir, autant mourir de façon valeureuse ${ }^{25}$.

Certaines chansons rendent compte de l'expérience de la traversée. La personnification des éléments naturels, tels que les vagues et le vent, est l'un des procédés stylistiques mobilisés à cette fin. Dans la chanson « Tri9 el-ghorba ${ }^{26}$ » (« Le chemin de l'exil »), le harrag qualifie les vagues de voleuses et de trâ̂tresses (خاين) et dit avoir été trompé par elles (الموج خدعني) 27. Afin de rendre compte des dangers de la traversée, les paroliers peuvent également insister sur les sensations ressenties par les « brûleurs » de frontières au moment de la traversée. C'est le cas, par exemple, de la chanson « Espoir perdu » dans laquelle Psyco-M rappe à propos de l'attente des harraga dans une maison isolée avant le départ. Il décrit le froid, la faim, la peur, ainsi que les rires qui cachent des larmes. Il fait le récit du départ à trois heures du matin et évoque ce que l'on ressent lorsqu'on est sur une embarcation cernée par la mer. Il compte le temps grâce au battement de son cœur. Lorsqu'il aperçoit des lumières, il a l'espoir d'être parvenu en Italie. Cependant, lorsque celles-ci se rapprochent, il comprend que ce sont les lumières d'un navire des gardes-frontières. La panique règne sur le bateau et le protagoniste tombe à l'eau. Les vagues sont hautes et il n'a rien pour se maintenir à la surface. Lorsqu'il comprend que sa vie va s'achever, il voit l'image de sa mère dans le ciel et repense aux «fils du quartier»

25. Cheb Rached, «Ya Roma » ("Ô Rome»), 2011.

26. "Dans la logique traditionnelle, el-ghorba est associé au "couchant", à l'"obscurité", à l'éloignement et à l'isolement (parmi les étrangers, donc à leur hostilité et à leur mépris), à l'exil, à la frayeur (celle que suscitent la nuit et le fait de se perdre dans une forêt ou une nature hostile), à l'égarement (par perte du sens de l'orientation), au malheur, etc. » (Sayad, 1999, p. 50). Dans ce texte, le mot ghorba est traduit par « exil ».
27. Bilel Al Achel, «Tri9 el-ghorba » ("Le chemin de l'exil »), 2012.

28. Psyco-M et Gadour, «Espoir perdu », 2011.

29. Inconnu, «Mezoued el-ghorba » (« Le mezoued de l'exil »), 2008. 
auxquels il demande pardon. Il meurt. Le débit du rappeur s'accélère au fur et à mesure et il simule à plusieurs reprises la perte de souffle ${ }^{28}$.

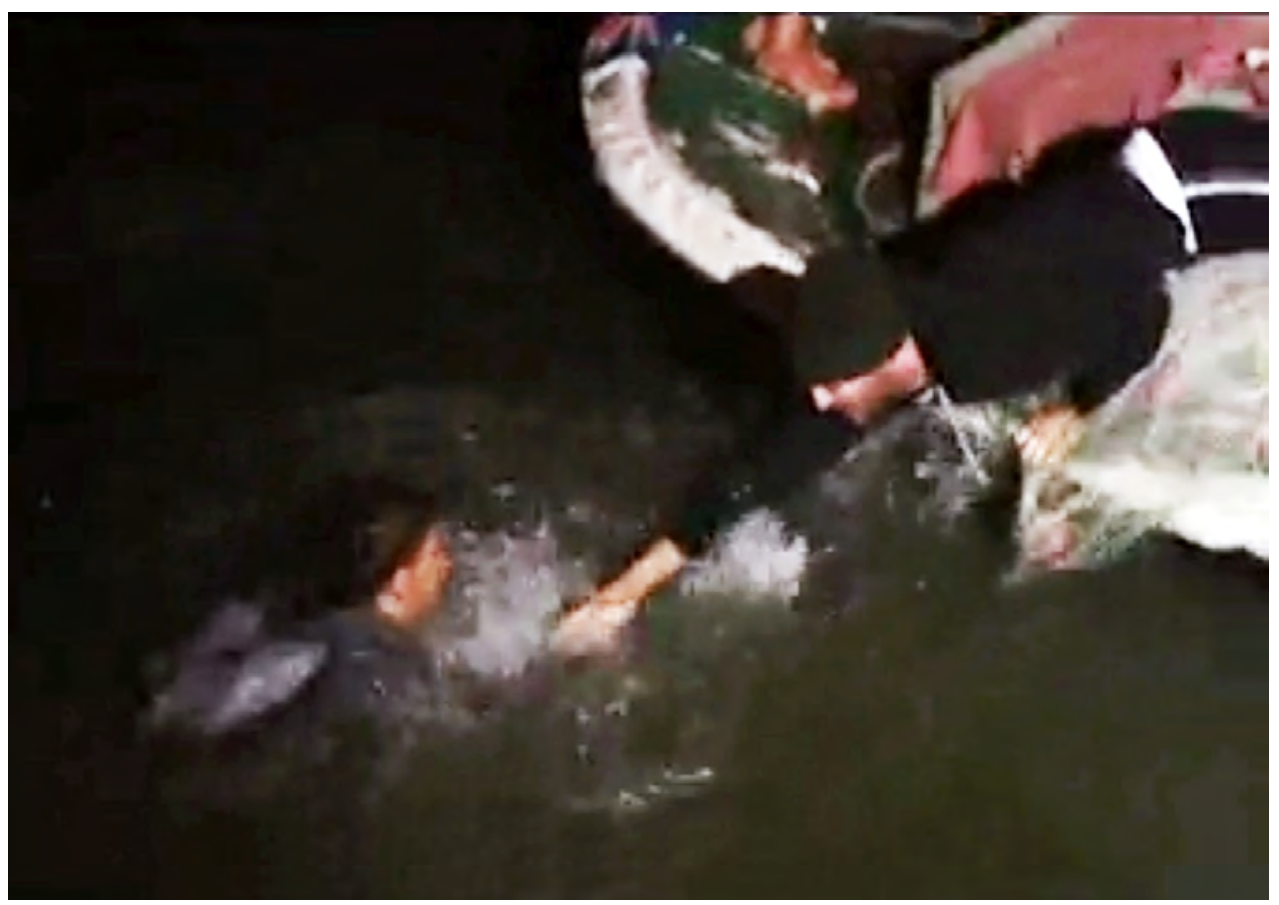

Du rap tunisien engagé. Sur cette séquence du clip vidéo de la chanson «El-7ar9a » de Sabrina et Rafik, nous voyons un bateau des harraga qui est repéré par les garde-côtes. L'un des passagers tombe dans la mer et se noie.

Photo prise en capture d'écran sur le compte Facebook de la rappeuse Sabrina : www.facebook.com/video.php?v=12250 9004496776\&set=vb.100002130329253\&type, 2016

Enfin, l'issue funeste de la traversée peut être racontée dans la perspective de ceux que les migrants laissent derrière eux. L'une des figures récurrentes des productions culturelles sur la harga est la mère éplorée. La chanson « Mezoued el-ghorba » («Le mezoued de l'exil ») est chantée dans la perspective de parents dont le fils est mort en Italie. La mère, dévastée, refuse que les gens viennent lui présenter leurs condoléances et leur affirme que son fils est toujours vivant et continue de lui parler. Par cette figure de la mère inconsolable, cette chanson évoque le deuil impossible des familles dont les enfants ont disparu ${ }^{29}$.

\section{La souffrance de l'exilé et des siens}

La souffrance des harraga et de leurs parents n'est pas uniquement liée à l'issue potentiellement funeste de l'aventure migratoire. Les thèmes de la désillusion et 
de la dureté de la vie en Europe sont également récurrents dans ce corpus. Dans sa chanson de mezoued, Ahmed El-Amri s'adresse directement à Lampedusa qu'il personnifie. Il reproche à l'île d'avoir fait pleurer sa mère, d'avoir fait de lui un vagabond, une blague, un triste spectacle qui s'offre aux yeux des Italiens. Les champs lexicaux utilisés pour rendre compte de la dureté du quotidien en Tunisie sont ici mobilisés pour décrire la vie à Lampedusa : larmes, douleur, amertume, tourment et angoisses ${ }^{30}$. Kaporal et Totorino écrivent dans la perspective d'un harrag qui vit dans la précarité et qui est contraint de voler pour pouvoir se nourrir. Il finit par trouver un travail comme vendeur de poisson, travaille jour et nuit, et mange ce qu'il trouve ${ }^{31}$. La représentation de l'émigré qui réussit en empruntant le chemin de la délinquance ou de la criminalité est récurrente au sein du corpus.

Dans « El-7ar9a », Mastaziano incarne un jeune qui souhaite « brûler » et Balti un ami qui souhaite l'en dissuader. Ce dernier argumente que seul un migrant sur mille réussit et que l'exil n'est pas le paradis. Il décrit des migrants traités comme des moutons sacrificiels, victimes du racisme et réduits à la mendicité. Les seules voies qui s'offrent à eux pour réussir sont la délinquance et la criminalité.

La souffrance de l'émigré n'est pas uniquement liée aux difficultés économiques auxquelles il est confronté en Europe. Le sentiment de solitude et de manque sont largement traités dans la chanson populaire tunisienne. Dans la chanson «El-ghorba 7keyet» («L'exil, des histoires »), Snappi et Mr Sabri mettent en avant l’isolement et le manque de solidarité qui s'ajoutent aux difficultés économiques.

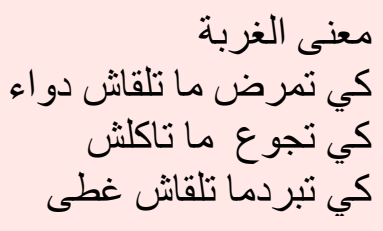

L'exil signifie :

Ne pas trouver de médicament quand tu tombes malade

Ne pas manger quand tu as faim

Ne pas trouver de couverture quand tu as froid ${ }^{32}$.

Les thèmes de la solitude et du manque sont également développés en recourant à la figure de la mère, déjà mentionnée. D'ailleurs, l'une des chansons du corpus est intitulée «Ya mima toua7chtek », ce qui signifie « Ô maman, tu

30. Ahmed El-Amri, "Lampedusa », 2012.

31. Kaporal et Totorino, "Direction Italy », 2011.
32. Snappi et Mr Sabri, «El-ghorba 7keyet», 2013.

33. Hamouda Artiste ft. El Capo, "Ya mima tou7chtek», 2013.
34. Kapo, Dadi BM et Ouzaier, «Tri9 I3mor», 2012.

35. Majhoul Mc, Mr M2H, Weld Cité $\mathrm{H}$, « El 7ar9a », 2011. 
me manques ${ }^{33} »$. Dans «Tri9 l3mor» (« Le chemin de la vie »), le harrag, une fois arrivé en Europe, chante :

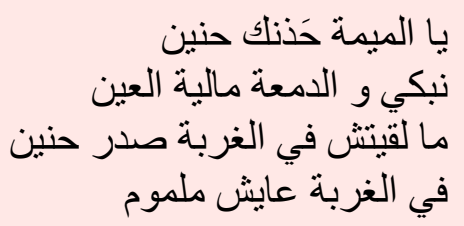

Ô maman, ton étreinte est tendre

Je pleure et les larmes emplissent mes yeux

Je n'ai pas trouvé dans l'exil une poitrine tendre

Dans l'exil, je vis délaissé34.

Le thème du manque est également abordé dans la perspective de ceux que les harraga laissent derrière eux. Dans « Mani mani ya Roma », Mohamed Taher décrit une mère qui a l'impression de perdre la raison et qui court derrière son fils au moment où celui-ci part. Dans «Limta traoua7? " («Quand reviendras-tu? »), le chanteur demande à «celui qui s'est absenté depuis tant d'années » (يا غايب ليك سني quand il retournera au pays. Les paroles insistent sur la douleur de sa famille, notamment de sa mère et de sa sœur qui ne cessent de pleurer et de se lamenter. Les rappeurs Majhoul Mc, Mr M2H et Weld Cité donnent la parole aux murs du quartier, qu'ils personnifient, afin de traiter de la même thématique. Ils évoquent un quartier déserté par ses habitants et dont les murs pleurent de douleur :

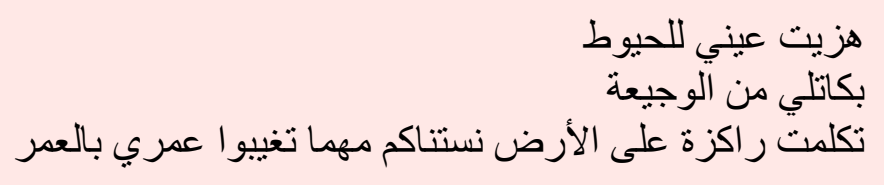

J'ai posé mes yeux sur les murs

Ils pleurent de douleur

Ils ont dit : nous sommes fixés sur la terre, nous vous attendrons même si vous partez plusieurs années ${ }^{35}$.

\section{Un voyage sous la protection de Dieu}

De nombreuses références à Dieu existent au sein du corpus. Le divin joue un rôle crucial dans la trajectoire de certains des harraga mis en scène dans les 
chansons. Ces protagonistes considèrent que leur avenir est déterminé par le maktoub ${ }^{36}$ et acceptent de s'en remettre à Dieu. Ils ont le sentiment d'évoluer sous le contrôle d'une puissance surnaturelle. Leur foi leur permet de donner du sens à certains événements qui surviennent lors de leur parcours migratoire ${ }^{37}$. Le migrant mis en scène dans la chanson de rap « $\mathrm{Ra} \mathrm{el}^{38}$ » demande à Dieu de ne pas l'abandonner, de l'aider à supporter sa situation et de le protéger. Le harrag qu'incarne Tarek Lagazaouali remet son destin entre les mains d'Allah :

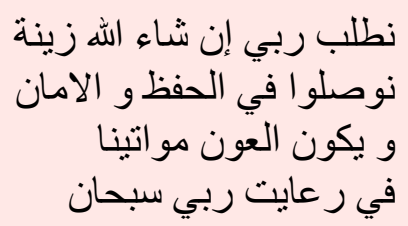

Je demande à Dieu, Inchallah, de faire en sorte que le voyage se passe bien On arrivera sain et sauf

Et le vent nous aidera

Par la protection de Dieu le glorieux ${ }^{39}$.

Si, la plupart du temps, c'est le harrag lui-même qui s'adresse à Dieu pour lui demander son aide, il fait parfois appel à ses parents, afin que ceux-ci fassent office d'intermédiaires auprès de lui. Dans « Lampedusa » de Mustapha Dellagi ${ }^{40}$, le harrag s'adresse à sa mère ${ }^{41}$ de la façon suivante :

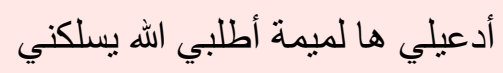

Prie pour moi, ma petite maman, et demande à Dieu de me sauver.

36. Le maktoub, ou mektoub, est une croyance selon laquelle tout ce qui advient est le produit de la volonté de Dieu. Maktoub signifie «ce qui est écrit » (du verbe "écrire ", كتب). En français, on le traduit souvent par «destin » ou «prédestination». La référence au maktoub fait partie des usages du quotidien, notamment en Tunisie où elle est très présente même chez des personnes qui n'ont pas de pratique religieuse.

37. Cette section présente plusieurs angles d'étude de la thèse de Simon
Mastrangelo (sous la direction de Monika Salzbrunn) qui est en cours de rédaction. Voir aussi Mastrangelo (à paraître).

38. Gregor et Sisko, «Ra7el »

(« Je m'en vais »), 2013.

39. Tarek Lagazaouli, «7ar9a », 2011.

40. Mustapha Dellagi,

"Lampedusa », 2012.

41. Certains harraga considèrent que leurs parents ont un meilleur comportement que le leur, parce qu'ils commettent moins d'interdits (notamment en rapport avec l'alcool et les drogues) et qu'ils ont une pratique religieuse plus régulière. Par conséquent, ils estiment que Dieu est plus enclin à écouter les demandes qui viennent de leurs parents (et en particulier de leur mère) que d'eux-mêmes.

42. Gregor et Sisko, «Ra7el » (« Je m'en vais »), 2013.

43. Gaddour, «El 7ar9a », 2011.

44. MC Riadh, Bayrem Batista, "tmnit nmchi el blad el ghir », 2013. 45. Cheb Rached, "Ya Roma " (Ô Rome), 2011. 
Le maktoub peut parfois servir d'argument pour convaincre ses parents du caractère inéluctable d'un départ vers l'Europe. Ceci est illustré dans une chanson où un harrag, souhaitant que sa mère accepte son choix de partir, lui déclare :

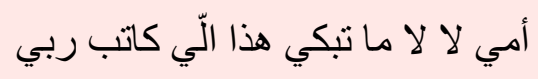

Maman, non, non, ne pleure pas ; c'est ce que Dieu a écrit ${ }^{42}$.

Dans «El-7ar9a $\mathrm{a}^{43} »$, Gaddour incarne tantôt un harrag qui explique à sa mère sa volonté de « brûler », tantôt un parent qui tente de dissuader son fils de partir. Tous les deux mobilisent des arguments religieux. Le harrag s'en remet à Dieu et lui demande de ne pas être indifférent à son sort. Ressentant le besoin d'obtenir l'accord de sa mère avant de partir, il lui dit que Dieu l'aidera si elle accepte son projet de départ. Quant à la mère, elle mobilise le maktoub et demande à son fils d'accepter sa situation au pays, malgré sa souffrance, car c'est ce que Dieu a voulu pour lui.

Le plus souvent, les textes font référence au maktoub pour exprimer un état de fait. Le destin étant inéluctable, il faut accepter son sort. Alors qu'il vit des moments difficiles à bord du bateau qui doit le mener vers l'Europe, le harrag dont parle le rappeur El Mister est persuadé d'être en train d'accomplir sa destinée. «C'est ce que Dieu a écrit pour moi » (ربي كتبلي), affirme-t-il. Se situer dans ce cadre procure une forme de réconfort puisque la destinée de chacun est décidée par un Dieu juste et miséricordieux. Même si le harrag a conscience du risque qu'il prend et qu'il craint la mort, le maktoub lui permet de rester serein face à l'éventualité d'une fin tragique, comme le proclament MC Riadh et Bayrem Batista :

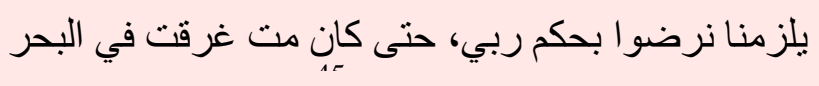

Que soit satisfaite la volonté de Dieu, même si je meurs noyé dans la mer ${ }^{44}$.

Si le plus souvent, les harraga sont mis en scène en train de demander l'aide de Dieu, ils expriment parfois leur reconnaissance envers Allah, comme dans «Ya Roma » («Ô Rome ») de Cheb Rached où le migrant déclare : « Nous sommes arrivés, Dieu nous est venu en aide ${ }^{45}$. » La réussite est ici interprétée comme le signe de la bienveillance divine. Arriver sain et sauf à destination, malgré les difficultés et les risques, est la preuve d'une validation divine. 


\section{La musique révélatrice des aspirations migratoires}

Les chansons consacrées à la harga sont-elles des objets politiques non identifiés, révélateurs de dynamiques sociales profondes dans un espace transnational rêvé et imaginé ? La lecture des paroles du corpus choisi ne fait aucun doute. L'analyse des textes a permis de déceler quatre thématiques en lien avec la harga. Le désir de «brûler » les frontières semble plus fort que tout. Outre l'imaginaire d'une vie plus facile en Europe, offrant la possibilité de soutenir sa famille, les chansons abordent aussi les conditions de vie éprouvées par la jeunesse tunisienne et leur désir de s'accomplir. L'imaginaire politique ne se réfère donc pas seulement à l'Europe, mais surtout aux conditions de vie dans une Tunisie instable, en crise économique, n'offrant que le chômage comme perspective, voire une mort lente. Cette vision de leur propre pays, liée à la motivation de s'accomplir en tant qu'hommes, de faire quelque chose plutôt que de se résigner, s'exprime dans les paroles des chansons. Ainsi, les harraga sont considérés comme des acteurs de leur destin, cherchant les moyens de dépasser les frontières de la forteresse Europe. Le voyage fantasmé et l'accueil en Europe évoqués à travers les paroles ne témoignent pas pour autant de naïveté. Au contraire, les migrants potentiels sont souvent décrits comme étant conscients des dangers de la traversée, de la souffrance, ainsi que de la déchirure qu'entraîne le choix de laisser les siens, en particulier leur mère, dans l'incertitude. Ainsi, l’image de la mère éplorée revient très souvent, au sens propre comme au sens figuré. Pour celle dont le fils est parvenu en Europe, la distance est source de souffrance. La mère du harrag disparu souffre de ne plus avoir de ses nouvelles et peine à concevoir sa mort.

Le harrag souffre de la séparation d'avec les siens, mais également parce qu'il est conscient qu'il les fait souffrir. La distance entre sa mère et lui peut symboliser la distance avec la mère patrie. En effet, le pays, et plus particulièrement le quartier de résidence en Tunisie, sont fréquemment évoqués avec nostalgie malgré le sentiment de ne pas pouvoir s'y accomplir. Une fois arrivé en Europe, le harrag est le plus souvent confronté à des conditions de vie particulièrement éprouvantes. Parfois, la stigmatisation et les difficultés auxquelles il est confronté amènent le harrag à envisager le retour. Cependant, dans la plupart des cas, ce n'est pas une raison d'abandonner son projet. La foi en Dieu et la croyance au maktoub permettent de résister aux peurs, de supporter les difficultés et de faire face au risque de mourir. Selon les paroles de certaines chansons, l'acte de partir s'effectue grâce à l'aide divine. Les sentiments d'exclusion et d'injustice peuvent se transformer en un discours d'émancipation, transformant des individus désespérés en acteurs de leur destin. Ces harraga se trouvent dans une tension entre une capacité d'agir et le sentiment de subir la situation, cherchant une issue afin d'accéder à la mobilité spatiale

46. Cette compréhension sera complétée par une analyse des mélodies et des mises en image (via des vidéo-clips ou des diaporamas que Heidrun Friese appelle "Ready-Mades ») dans des publications ultérieures (cf. Friese, 2012, et Salzbrunn, 2012, sur les aspects visuels). 
et sociale. Les acteurs, dont parlent ces chansons maîtrisent non seulement symboliquement le monde (Arnaud, 2014), mais retournent aussi le stigmate de manière concrète en décidant de partir. L'analyse des paroles de rap et du mezoued permet ainsi de comprendre des dynamiques sociales profondes ${ }^{46}$. Sans pouvoir être classées dans la musique qui devient révolutionnaire par sa réception (Peddie, 2012, p. XIII), les paroles contiennent un potentiel de subversion important : celui de voter avec ses pieds. 


\section{Bibliographie}

Agier, M. (2008), Gérer les indésirables. Des camps de réfugiés au gouvernement humanitaire, Paris, Flammarion.

Arnaud, L. (2014), "Les "dominés" peuvent-ils créer? L'action culturelle des "minorités ethniques", entre mythe et réalités", SociologieS, dossier "Diversification artistique et politiques culturelles", 25 septembre.

Auzanneau, M. (2001), «Identités africaines: le rap comme lieu d'expression ", Cahiers d'études africaines, vol. XLI, n 163-164, p. 711-734.

Bensaâd, A. (2009), Le Maghreb à l'épreuve des migrations subsahariennes: immigration sur émigration, Paris, Karthala.

Biddle, I. (2012), "Introduction", in I. Biddle, Music and Identity Politics, Newcastle, Newcastle University, p. XI-XXIV.

Boubakri, H. (2013), « Migrations internationales et révolution en Tunisie », Migration Policy Center.

Canut, C., Sow, A. (2014), " Les voix de la migration. Discours, récits et productions artistiques ", Cahiers d'études africaines, $n^{\circ}$ 213-214, p. 9-25

Di Bartolomeo, A., Fakhoury, T., Perrin, D. (2010), "Migration Profile. Tunisia", CARIM, EUI.

Düvell, F. (2008), "Clandestine Migration in Europe", Social Science Information, vol. XLVII, $n^{\circ} 4$, p. 479-497.

El Qadim, N. (2013), « Négocier l'asymétrie : les politiques extérieures européennes au regard des relations entre acteurs marocains et européens du gouvernement des migrations ", thèse de science politique, Sciences Po.

Friese, H. (2012), "Y'al babour, y'a mon amour. Raï-Rap und undokumentierte Mobilität", M. Dietrich, M. Seeliger, Deutscher Gangsta-Rap, Bielefeld, Transcript Verlag, p. 231-284.
Friese, H. (2015), "Transnational Mobilities, Digital Media and Cultural Resources", in A. Lichtenberger, C. von Rüden (dir.), Multiple Mediterranean Realities. Current Approaches to Spaces, Resources, and Connectivities, Munich, Fink Verlag, p. 273-311.

Frikha, Z. (2009), «Appropriation locale d'un phénomène global. Le rap tunisien ", mémoire de master en anthropologie sociale et culturelle, sous la direction d'Imed Melliti, université de Tunis, El Manar.

Gana, N. (2012), "Rap and Revolt in the Arab World", Social Text, vol. XXX, n 4, p. 25-53.

Hibou, B. (2005), «Économie politique de la répression : le cas de la Tunisie ", Raisons politiques, $n^{\circ} 20$, p. 9-36.

Hibou, B. (2011), « Tunisie. Économie politique et morale d'un mouvement social ", Politique africaine, $n^{\circ} 121$, p. 5-22.

Hollifield, J. (1992), Immigrants Markets and States. The Political Economy of Postwar Europe, Cambridge, Harvard University Press.

Labdelaoui, H. (2009), « Harga où la forme actuelle de l'émigration irrégulière des Algériens ", CARIM, $n^{\circ} 18$.

Mabrouk, M. (2010), Voiles et sel : culture, foyers et organisation de la migration clandestine en Tunisie, Tunis, Sahar.

Martin, D.-C. (2003), Sur la piste des OPNI (Objets politiques non identifiés), Paris, Karthala.

Martiniello, M., Lafleur, J.-M. (2008), "Ethnic Minorities. Cultura and Artistic Practices as Forms of Political Expression. A Review of the Literature and a Theoretical Discussion on Music", Journal of Ethnic and Minority Studies, vol. XXXVIII, nº 8, p. 1191-1215.

Mastrangelo, S. (à paraître), "Représentations de parcours migratoires de harraga tunisiens Lecture à la lumière de la foi en Dieu », in N. Ortar, M. Salzbrunn, M. Stock, Mobilité-Migration.
Propositions épistémologiques pour appréhender les déplacements humains.

Mattern, M. (1998), Acting in Concert. Music, Community, and Political Action, New Brunswick, Rutgers University Press.

Migration Policy Centre (2013), Tunisia Migration Profile, Florence, EUI, RSCAS

Migreurop (2012), Atlas des migrants en Europe. Géographie critique des politiques migratoires, Paris, Armand Colin.

Natter, K. (2014), "The Formation of Morocco's Policy Towards Irregular Migration (2000-2007), Political Rationale and Policy Processes ", International Migration, vol. LII, n 52 p. 15-28.

Peddie, I. (2012), Music and Protest, Farnham, Ashgate Publishing.

Peraldi, M. et al. (2014), «S'arracher: la harraga des mineurs en Algérie », in M. Peraldi, Les Mineurs migrants non accompagnés : un défi pour les pays européens, Paris, Karthala, p. 143-176.

Rouis, S. (2008), « La migration irrégulière en Tunisie. Modes d'approches et techniques de recherches ", atelier sur les migrations africaines, "Les recherches sur les migrations africaines : méthodes et méthodologies ", 26-29 novembre, Maroc, Rabat.

\section{Saadane, $H_{\text {., Fluhr, }}$., Guidère,} M. (2013), « La reconnaissance automatiques des dialectes arabes à l'écrit », colloque international "Quelle place pour la langue arabe aujourd'hui ? », 18-20 décembre, Algérie, Alger.

Salzbrunn, M. (2008), "World Society, Transnationalism and 'Champs Migratoires'. Reflections on German Anglo-Saxon and French Academic Debates", in R. Gabriel Anghel, E. Gerhartz, G. Rescher, M. Salzbrunn, The Making of World Society. Perspectives from Transnational Research, Bielefeld, Transcript-Verlag, p. 75-100. 
Salzbrunn, M. (2012),

"Undocumented Mobility (TunisiaSwitzerland) and Digital-Cultural Resources after the 'Arab Spring'", projet financé par la Fondation nationale suisse pour la recherche.

Salzbrunn, M., Mastrangelo, S. (2014), "Digital Humanities Empowering through Arts and Music. Tunisian Representations of Europe through Music and Video Clips", Digital Humanities Conference, Lausanne, EPFL.

Salzbrunn, M., Mastrangelo, S., Souiah, F. (à paraître), « Migrations non-documentées et imaginaires sur Internet. Le cas des harraga tunisiens ", communication aux $12^{\text {es }}$ Journées européennes de Galatasaray organisées par le CREDE, «Visions croisées autour des frontières européennes: mobilité, sécurité et frontières ».

\section{Liste des chansons du corpus:}

Achraf, "Ra7 wlidi » (" Mon fils est parti »), 2012, 7' 24".

Ahmed El-Amri, "Lampedusa », 2012, 7' 35".

AKM, "9assed rabi » (" Je m'en remets à Dieu »), 2012, 2' 55".

Ali Dji et Amine, « Machi » (" Je pars »), 2015, 5' 24".

Balti et Mastaziano, "El-7ar9a » ("La harga "), 2011, 6' 44".

Balti et Samir Loussif, " Mchaou » ("Ils sont partis "), 2011, 5' 58".

Baster et Malek, "Wa3di » (" Ma promesse »), 2012, 4' 57".

Bilel Al Achel, "Tri9 el-ghorba » ("Le chemin de l'exil »), 2012, 9' 02".

Cheb Rached, "Ya Roma "

("Ô Rome »), 2011, 6' 54".

CTH Familia, "Barcha dmou3 " ("Beaucoup de larmes »), 2008, 4' 11".

DJ Costa, "Ya tayara " ("Ô avion »), 2011, 4' 56".
Shannahan, D.S., Hussain, Q. (2010), "Rap on 'L'Avenue'. Islam, Aesthetics, Authenticity and Masculinities in the Tunisian Rap Scene", Contemporary Islam, vol. V, $n^{\circ} 1$, p. 37-58

Souiah, F. (2011), « Musique populaire et imaginaire migratoire en Algérie », Diversité, n¹64, p. 27-33.

Souiah, F. (2013), « Les politiques migratoires restrictives : une fabrique de harraga ", Hommes \& Migrations, vol. MCCCIV, n4, p. 95-101.

Souiah, F. (2014), "Les "brûleurs" de frontières dans les productions culturelles ", "Les harraga en Algérie. Émigration et contestation ", thèse de science politique, Sciences Po, p. 263-332.

DJ Costa, "Hani machi » (" Je pars »), 2011, 3' 39".

DJ Costa, "Na3cha9 el-ghorba » ("Je veux m'exiler »), 2013, 5' 39".

DJ Costa, «El 7oudoud» (« Les frontières »), 2013, 3' 31".

EI Mister, « Vue noire », 2012, 5' 25".

\section{Familya Music and Maklay} Music, "Kharej men Tounes» (" Je quitte la Tunisie »), 2013, 3' 14".

F'iros raggae man, "Yammi » («Ô maman »), 2012,4' 05".

Gaddour, «El-7ar9a » ("La harga »), 2011, 7' 20".

Gregor et Sisko, "Ra7el » ("Je m'en vais »), 2013, 3' 46".

Hamouda Artiste et EI Capo, «Ya mima, toua7chtek " ("Ô maman, tu me manques »), 2013, 4' 17".

\section{Houssem Ben Romdhane Ft}

Linterboys, "Sou9 bina ya rayes » ( $O$ O capitaine, emmène-nous »), 2013, 4' 37".

\section{Jocker Boss et Lalpadzi,}

"Berrani » ("Étranger »), 2013, 2'43".
Stapley, K. (2006), "Mizwid. An Urban Music With Rural Roots", Journal of Ethnic and Migration Studies, vol. XXXII, n² 2, p. 243-256.

Virolle, M. (1995), La Chanson raï: de l'Algérie profonde à la scène internationale, Paris, Karthala.

Wihtol de Wenden, C. (2010 et 2013), La Question migratoire au $x x I^{e}$ siècle: migrants, réfugiés et relations internationales, Paris, Les Presses de Sciences Po.

Jouini Skander, «El 7ar9a» ("La harga »), 2013, 6' 48".

Kapo, Dadi BM, Ouzaier, «Tri9 la3mor" ("Le chemin de la vie »), 2012, 3' 32".

Kaporal et Totorino, "Direction Italy », 2011, 4' 03".

\section{Klay BBJ et Hamzaoui Med}

Amine, "Ritouchi » ("Avez-vous vu ?»), 2011, 4' 12".

KingBoy et Safwen, "T3ddit el7doud » ("J'ai traversé les frontières »), 2011, 4' 06".

Lamjed El-Gafsi, "Fi Italia 5allani » (“ II m'a laissé en Italie »), 2012, 8' 15".

L'artisto et I'inspecteur, " Ya 7ara9 choufli blaça " ( Ô passeur, trouve moi une place »), 2012, 4' 36".

Lotfi El-Weghémi, " Limta traoua7? " («Quand reviendras-tu ?»), 2010, 5' 33".

Majhoul Mc, Mr M2H,

Weld Cité H, "El-7ar9a »

(«La harga »), 2011, 5' 36".

Mascott, "Msafer » (" Je voyage »), 2011, 5' 36". 
MCH et Cheb Kais, "Ya b7ar» ("Ô mer »), 2011, 4' 59".

\section{Riadh, Bayrem Batista,}

"Tmnit nmchi lebled el-ghir» (" Je veux partir au pays des autres »), 2011, 3' 53".

MC Saber et Fils2Bled, "Yezni » ("Ça suffit »), 2012, 7' 16".

MC Volcano, "Ghodwa 7ar9a " ("Demain, harga »), 2009, 3' 33".

Mohamed Taher, "Mani mani ya Roma » (" Ô Rome »), 2009, 6' 06".

Mr. Mustapha, «7ar9a » ("Harga »), 2010, 6' 06".

Mustapha Dellagi, "Lampedusa ", 2012, 13' 57".
Nabil Louhichi, «Yamma »

("Ô maman »), 2011, 10' 10".

Nabil Louhichi, "El-ghorba 7kayet » ("L'exil, des histoires »), 2011, 8' 54".

Nabil Louhichi, «Paris », 2012, 7' 43".

Nabil Louhichi, «Ya Roma », 2012, 7'43".

Prince-M, MhB et TaBarkano, " Je veux quitter le bled ", 2012, 3' 29".

Psyco M et Gaddour, "Espoir perdu », 2011, 6' 35".

Riadh Bakkar, " 7yeti nersmha bdem3a » ("Je dessine ma vie avec une larme »), 2011, 10' 59".
Sabrina et Rafik, "El-7ar9a », 2008, 4' 08".

Samir Loussif, "Ya mima, fil ghorba », 2013, 5' 57".

Sincero, « Mr le Président », 2011, 4' 37".

Snappi et Mr Sabri, «El-ghorba 7keyet » ("L'exil, des histoires »), 2013, 4' 03".

T-Gang, "Yamma » (" Maman »), 2012, 4' 29".

Tarek Lagazouali, « 7ar9a » ("Harga »), 2011, 5' 53".

Inconnu, « Mezoued el-ghorba » ("Mezoued de l'exil »), 2008, 8' 49". 